PPPL-2754

UC-426

PPPL-2754

DEVELOPMENT OF SMALL SCALE SOFT X-RAY LASERS

BY

D. KIM, C.H. SKINNER, D. VOORHEES, AND S. SUCKEWER

May 1991
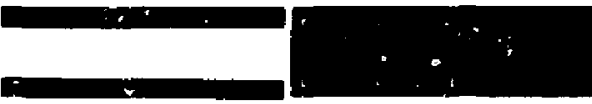

PFinut TON

DLAfma murace

LABomatopr

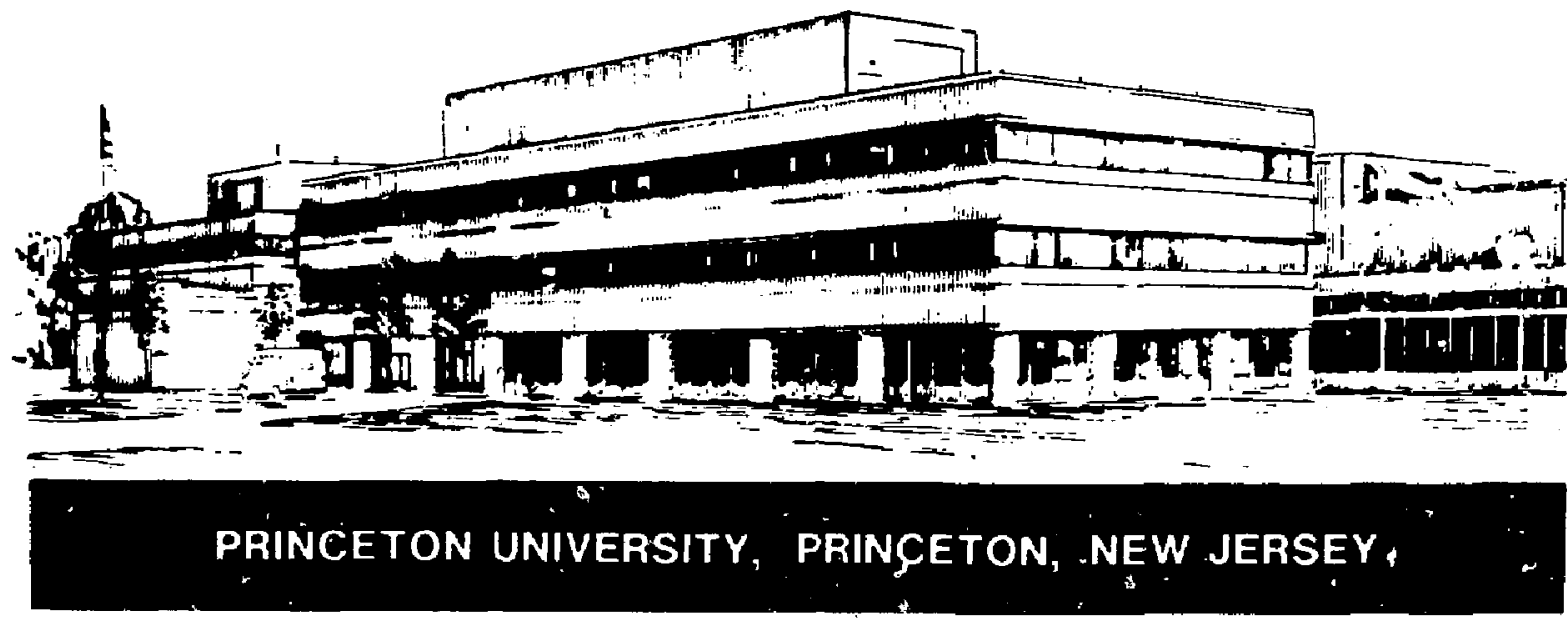




\section{NOTICE}

This roport was prepared as an account of work sponsored by an agency of the United States Government. Neither the United States Government nor any agency thereof, nor any of their employees, makes any warranty, express or implied, or assumes any legal liability or responsibility for the accuracy, completeness, or usefulness of any intormation, apparatus, product, or process disclosed, or represents that its use would not infringe privately owned rights. Reference herein to any specific commercial produce, process, or service by trade name, trademark, manufacturer, or otherwise, does not necessarily constitute or imply its endorsement, recommendation, or favoring by the United States Government or any agency thereof. The views and opinions of authors expressed herein do not necessanily state cr reflect those of the United States Government or any agency thereof.

\section{NOTICE}

This report has been reproduced directly from the best available copy.

Available to DOE and DOE contractors from the:

Office of Scientific and Technical Information

P.O. Box 62

Oak Ridge, TN 37831 ;

Prices available trom (615) 576-8401.

Available to the public from the:

National Technical Information Service

U.S. Department of Commerce 5285 Port Royal Road Springfield, Virginia 22161 $703-497-4650$ 


\title{
DEVELOPMENT OF SMALL SCALE SOFT X-RAY LASERS
}

PPPL- -2754

\author{
D. Kim*, C.H. Skinner, D. Voorhees, and S. Suckewer* \\ Princeton University, Plasma Physies Laboratory \\ P.O.B. 451, Princeton N.J. 08543
}

\begin{abstract}
At present rapid progress is being made in the application of soft $x$-ray lasers io fields such as microscopy and microlithography. A critical factor in the range of suitable applications is the scale and hence cost of the soft $x$-ray lasers. At Princeton, gain at $182 A$ has been obtained with relatively low pump laser energies (as low as $6 \mathrm{~J}$ ) in a 'portable' small-scale soft $x$-ray laser system. We will also discuss aspects of data interpretation and pitfalls to be avoided in measurements of gain in such systems.
\end{abstract}

\section{INTRODUCTION}

When the first laser was demonstrated by Maiman in 1960, the directionality and brightness of these new sources caught the public imagination but they were slow to find applications. In fact, there was an extended period when they were known as "solutions looking for a problem." Today of course 30 years later the situation is very different with laser scanners in widespread use in shops and compact disk players and even in dentists' offices.

It is now six years since the furst demonjtration of high gain in the soft $\mathrm{x}$-ray region, and we are now in a period between demonstrations of gain in different systems and the acceptance of $x$-ray lasers as practical and useful devices. Of course research on soft $x$-ray lasers is expensive and if it was going to take 20 years or so for applications to emerge then the funding prospects might indeed be limiter'. Fortunately the development of applications of soft $x$-ray lasers is in progress and it is very important to demonstrate as soon as possible that soft $x$-ray lasers are not just exotic and expensive specialized laboratory phenomena but can be practica! tools with imporant applications.

This work is part of a larger effort at Princeton of which an overview is given in the paper by Suckewer(1990). Work on the two laser approach aimed at generating lasing in the $10 \mathrm{~A}$ region is reported by Tighe(1990). This paper is about the work at Princeton to develop small scale soft $X$-ray lasers for applications. A review of the application of soft $x$-ray lasers to $x$ ray microscopy is given in this proceedings (Suckewer 1990) and in (Skinner 1990a).

\section{SMALL SCALE SOFT X-RAY LASERS}

The original recombination pumped $182 \AA$ laser at Princeton was based on a magnetically confined $\mathrm{CO}_{2}$ laser produced plasma. While this laser continues to be used for applications development, efforts have begun to improve its performance by increasing the energy with additional amplifiers. To this end we are developing amplifiers pumped with a line focussed neodymium laser. Initial results yielded a gain of $8 \mathrm{~cm}^{-1}$ on the CVI $182 \AA$ transition using a pump laser energy of only 15J on target (Kim 1989a). In other experiments (Skinner 1990b) gain of $4.5 \mathrm{~cm}^{-1}$ at $182 \mathrm{~A}$ was obtained with only $6 \mathrm{~J}$ of driving laser energy. This is a remarkably low pump energy and has very favorable implications for the future widespread use of these devices. The target length in this case was limited by the diameter of access ports

- also at Dept. of Mechanical and Aerospace Engineering. Princeton Univirsity
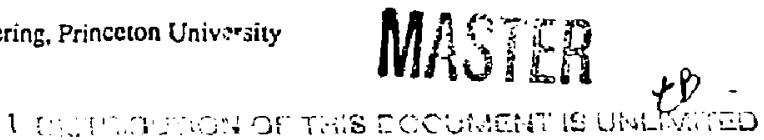
in the magnet. Aluhough the gain was high, the relatively short target length limited the gainlength to $G L=2-3$ and hence the output intensity of the stimulated emission was only several times that of the spontaneous emission. Clearly, from the point of view of applications we would like to be in the region of $G L=5$ or more where the output is orders of magnitude higher than spontaneous emission. Practical obstacles such as plasma uniformity and refraction of the stimulated emission beam need to be overcome in order to maintain gain over larger lengths.

To address theses issues, a new target chamber was installed outside the solenoidal magnet which enabled longer length targets to be used. In order to maximize the gain and confune a plasma, the interaction region of a target was surrounded by a miniature chamber with stainless steei walls (see Figure 1). Gain is generated by recombination after the plasma is first ionized by the drive laser. The recomtination rate and hence gain generated depend strongly on the cooling rate. The stainless steel walls confine the plasma and provide additional cooling both by radiation losses from iron impurities in the plasma and by thermal conduction.

A $67-c \mathrm{~m}$ focal-length spherical lens and two cylindrical lenses were operated to produce a $\leq 100 \mu \mathrm{m} \times 11 \mathrm{~mm}$ line-focus on a length-varying cylindrical target. A square apodiser was used to control the Nd laser beam cross section to improve the uniformity of the line focus. The target lengths used in this experiment were $2.5,5.0$, and $7.5 \mathrm{~mm}$ A $0.2 \times 2 \mathrm{~mm}$ slot in a mask located $10 \mathrm{~cm}$ away from the carget in the axial direction, selected a limited spatial region which was viewod by an axial soft $x$-ray spectrometer equipped with a multichannel detector. In the experiments the slot was placed in such a way that it selected a spatial region $0.4-0.6$ mm from the target surface.

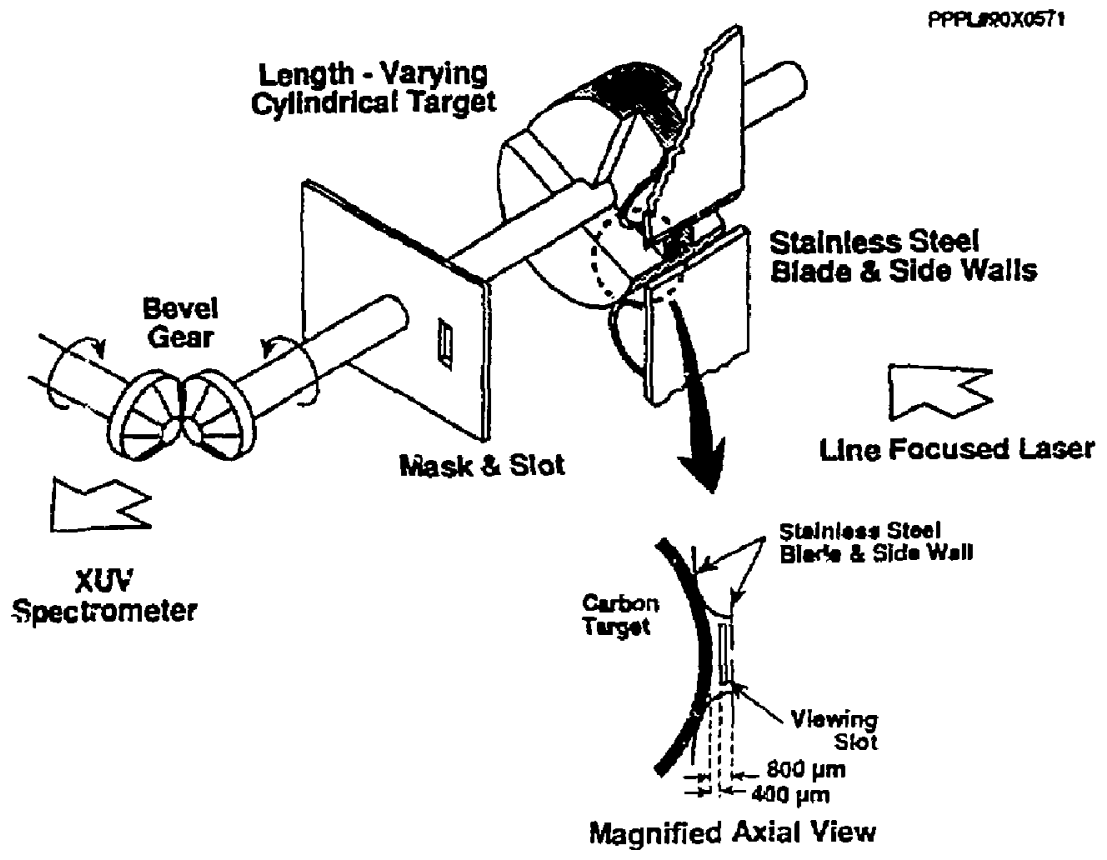

Fig. I The rotatable target system. The Nd laser enters through a stainless steel slot $400 \mu \mathrm{m}$ wide. The plasma is surrounded by flexible stainless steel walls to proride additional cooling and the view of the axial spectrometer is restricted to the region shown in the lower axial view. Part of the upper blade and side wall has been cut away in the drawing to display the target. 


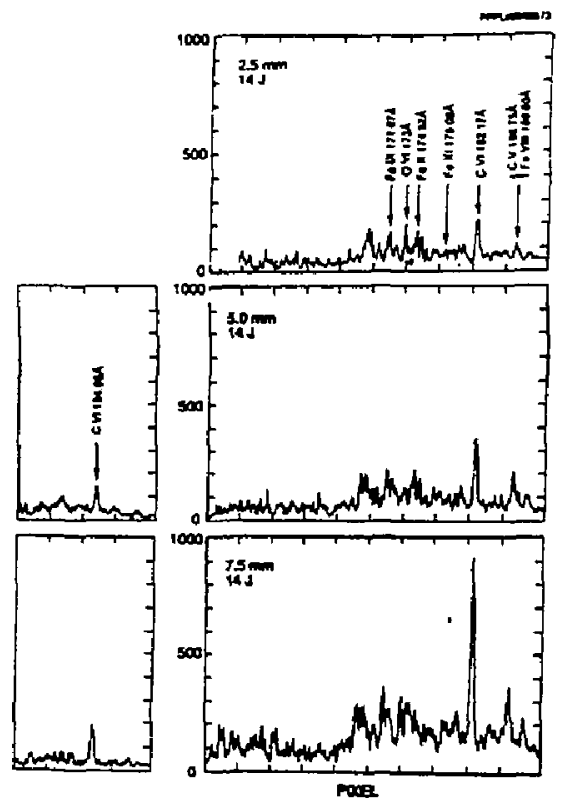

Fig. 2 Spectra obtained with $14 \mathrm{~J}$ laser energy from carbon plasmas of length: (a) $2.5 \mathrm{~mm}$, (b) $5 \mathrm{~mm}$ and (c) $7.5 \mathrm{~mm}$.

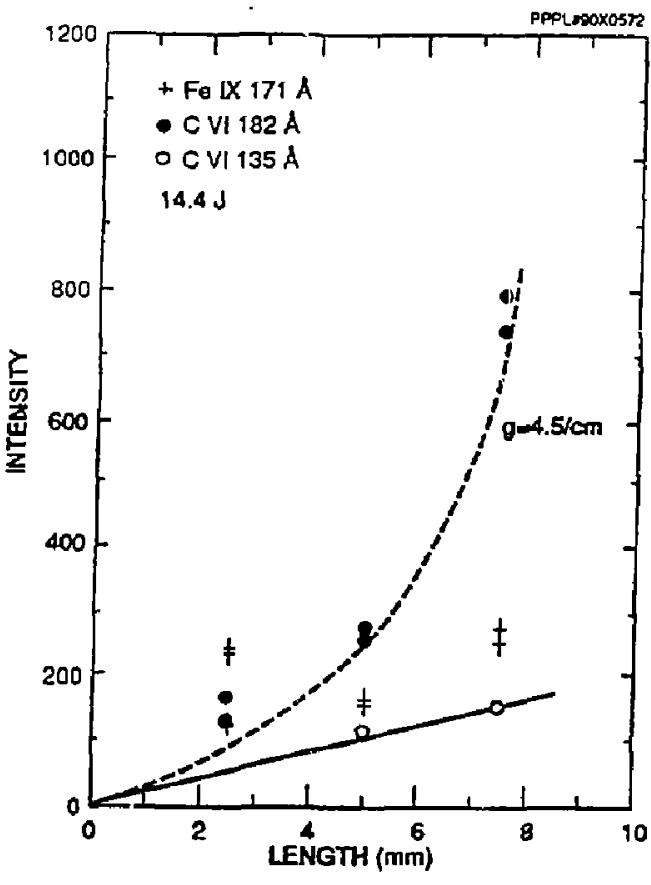

Fig. 3 Inrensities of the CVI $182 \AA$ and $135 \dot{A}$ lines versus plasma length and (dashed line) a least squares fit to the gain equation (eqn. 1) with a gain of $4.5 \mathrm{~cm}^{-1}$ for the $182 \AA$ line.

Preliminary results, obtained with a 14J, 3nsec laser pulse are shown in Figures. 2 and 3. A conqubution from a FeXI 182.17 $A$ line has been subtracted from the data. The Fe contribution was estimated from the intensity of the FeXI 178.1A line. This line shares the same upper level $\left(3 p^{3} 3 d^{3} D_{2}^{\circ}\right.$ ) with FeXI $182.17 \AA$ and the relative intensity of these two lines is given by Kelly (1987). The CVI $182.17 \AA$ lin - (3-2 transition) increased non-linearly while the CVI $134.95 \AA$ and some other lines increased linearly as expected from optically thin spontaneous emission from a homogeneous plasma of length equal to the length of the target. This was a clear indication of gain on the $182.17 \AA$ line. The difference in the length dependence of the $182.17 \AA$ and $134.95 \AA$ lines here is very important. The data were fitted by a nonlinear regression model which performed a least-square fit of the data to the relation (Linford 1974) :

$$
I(L) \propto \frac{(\exp (C L)-1)^{3 / 2}}{(G L \times \exp (G L))^{1 / 2}}
$$

This describes the ourpur intensity of a Doppler-broadened, homogeneous source of amplified spontaneous emission of gain-length product GL. The fit yielded a value of the gain of 4.5 $\mathrm{cm}^{-1}$ on the CVI 182.17 $\mathrm{A}$ line. These results at remarkably low driving laser energy augur well for the commercial availability in the near future of relatively inexpensive soft $x$-ray lasers for a variety of novel applications. 


\section{EXPERIMENTS ON ALUMINUM PLASMAS, ASPECTS OF DATA INTERPRETATION}

A new target chamber has been constructed with improved access for the drive laser so that plasmas of length of $1 \mathrm{~cm}$ or more could be produced (Skinner 1990b). Because of concen about deviations of the beam due to refraction, this system also had much more flexibility for positioning and angular adjustment of the target and detector. In this section we present some results from this system showing non-linear increase of intensity with length of ALX and AlXI lines in an aluminum plasma. The lithium sequence ions such as AlXI were first used in soft $x$-ray laser development by Jaegle (1990); however the present work on aluminum plasmas ereated with a low energy Nd laser was primarily stimulated by the surprising results of Hara (1989) indicating gain on alnost all AlX and AlXI lines observed. It was a simple task to repeat the experiments of Hara et. al. by changing the target material to aluminum.

A Nd glass laser, operated at $6 \mathrm{~J}$ or $12 \mathrm{~J}$, was brought to a line focus by a combination of 4 lenses. Two spherical lenses with a combined focal length of $60 \mathrm{~cm}$ and two cylindrical lenses produced a sharp line focus $12 \mathrm{~mm}$ long with a width of $50 \mu \mathrm{m}$ (FWHM) on a rotatable aluminum target with sectors of differing length $(2,6,10 \mathrm{~mm})$. Axial emission was detected by a soft X-ray multichannel spectrometer "SOXMOS" (Schwob 1989) SOXMOS was attached to a rotatable arm pivoted under the target so that the angle it viewed could be varied by $\pm 2^{\circ}$ with respect to the target. The target assembly was on a platform that could also be rotated $\pm 2^{\circ}$ around a vertical axis. By combining the two motions, emission over a $\pm 4^{\circ}$ axial range in the horizontal plane could be recorded. This system was designed to aliow the most precise alignment of the target with respect to the spectrometer and also enable the detection of a stimulated soft $X$-ray beam that had been deviated from the nominal axial direction by refraction in the plasma. A slot with open area $3 \mathrm{~mm}$ high and $0.35 \mathrm{~mm}$ wide was placed on axis $4 \mathrm{~cm}$ from the targat to limit the view of the spectrometer. The position of the slot could be adjusted to view regions of the plasma ar different distances from the target surface.

In the experiment a search for gain was performed by varying the experimental parameters (including the target length) and looking for conditions in which the intensity of candidate lines increased with length at a rate that was faster than linear. A faster-than-linear rise of intensity with length is commonly regarded as conclusive evidence for stimulated emission.

Some very interesting results emerged very quickly in the experiments. At $6 \mathrm{~J}$ drive laser energy a very dramatic increase in the AlX and AlXI lines was seen with the $10 \mathrm{~mm}$ target as compared to $2 \mathrm{~mm}$, while the AlIV and OVI lines show a sub-linear increase. The data could be ritted to the Linford (1974) relation with a gain of $4 / \mathrm{cm}$ for the Al XI $154 \AA$ line and the $3.3 / \mathrm{cm}$ for ALX 177A. We repeated the experiments at $12 \mathrm{~J}$ and observedan even more dramatic effect (Figures 4 and 5).

All the AlXI and AlX lines observed show an non-linear increase with length in an almost perfect fit to the Linford relation for gain. For instance the AlXI $141 \bar{A}$ ( $3 s-4 p$ ) intensity increases by a factor of $x 50$ from $2 \mathrm{~mm}$ to $10 \mathrm{~mm}$ and is an excellent fit to the gain equation for

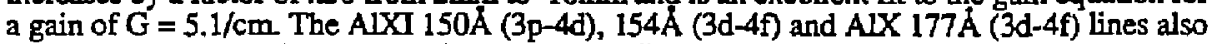
show a length dependence which is a very close fit to the gain equation at comparable values of gain. Similar results were obtained for the AlXI 105.7 $(3 \mathrm{~d}-5 \mathrm{f})$ and $103.8 \AA$ (5d-3p) lines. 

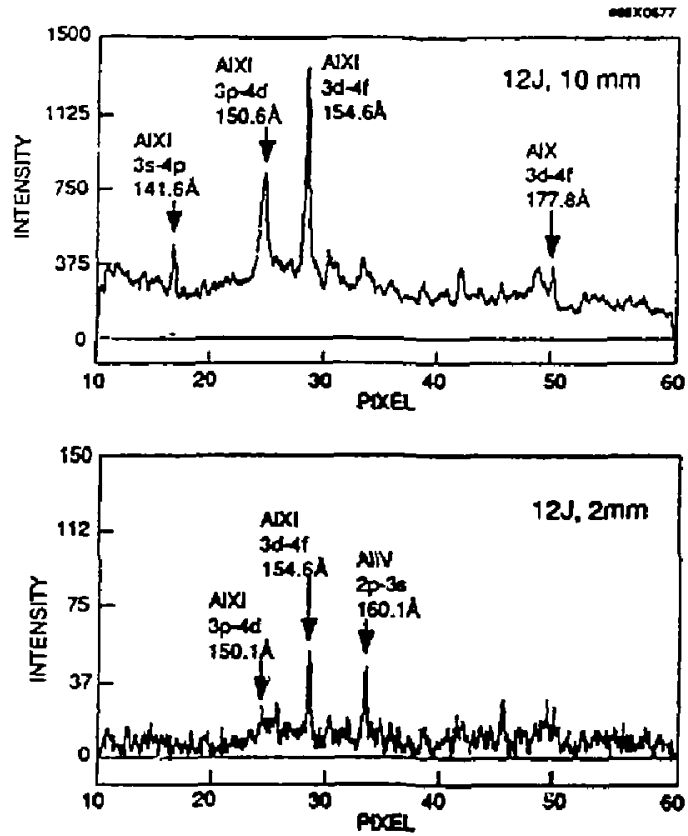

Fig. 4 Spectra obtained at $12 \mathrm{~J}$ laser energy with target length $2 \mathrm{~mm}$ and $10 \mathrm{~mm}$. Note the $\times 10$ scale change from the $2 \mathrm{~mm}$ to $10 \mathrm{~mm}$ graph

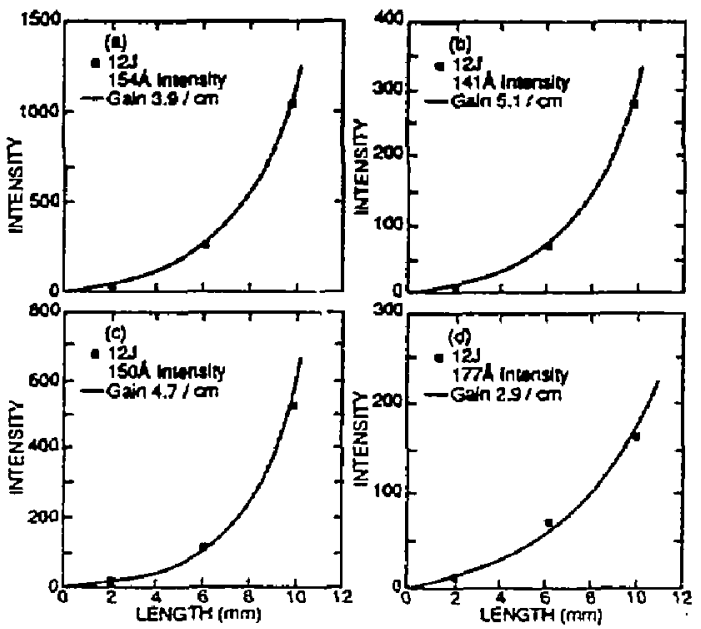

Fig. 5 Dependence of the AlXI 141 $\AA, 150 \AA, 154 \AA$ and $A 1 X 177 \AA$ axial line intensity on length. The line represents a theoretical fit of the experimental points to the gain equation (Eq. (1) in the text). 


\section{DATA ANALYSIS}

There are some unexpected features to the data in Figures 4 and 5. First of all, every ALX and AlXI line observed, without exception, showed a non-linear increase with length. In general the gain coefficient depends on the factors shown in Eq.(2):

$$
G=\frac{1}{8 \pi c} \frac{\lambda^{4}}{\Delta \lambda} g_{i} A_{i k}\left\{\frac{N_{i}}{g_{i}}-\frac{N_{k}}{g_{k}}\right\}
$$

Here $G$ is the gain coefficient, and $\lambda$ the wavelength. $g$ is the statistical weight, $A_{i k}$ the radiative transition probability and $N$ the population of the upper level $i$ and lower level $k$. The highest gain was expected on the 3-4 transition with the largest gA value (3d-4f at $154 \AA$ ); however, the dara shows high gain on all the AIXI and AlX lines observed, similarly to Hara et. al. (1989). Particularly surprising was the strong increase apparent on the AIXI $141 \mathrm{~A}$ line which has a gA value much lower than the $150 \AA$ and $154 \AA$ transitions. In fact in previous work in magnetically confined, $\mathrm{CO}_{2}$ laser-produced systems (Kim 1989b), where the ALXI $154 \AA$ transition exhibited stimulated emission; the $141 \AA$ line emission was solely spontaneous. In the present experiment the time history observed with a streak camera showed no difference between the time evolution at $154 \AA$ and the continum background at $162 \AA$ and is not delayed as might be expected in a recombination system. Another unexpected feadure is the apparent 'gain' in the background continuum emission, from 5-10 counts at $2 \mathrm{~mm}$ to $\sim 200$ counts at $10 \mathrm{~mm}$, an increase of -30 for a factor of 5 change in length. All these features raised concerns about the homogeneity of the plasma along its length. Specifically, were the level populations in the region of the $2 \mathrm{~mm}$ plasma viewed by the spectrometer identical to the conditions in the $10 \mathrm{~mm}$ section? The 2, 6 and $10 \mathrm{~mm}$ sections shared a common boundary on the spectrometer end of the target wheel. To test if the plasma was homogeneous, a varget was built with the $2 \mathrm{~mm}$ sections on both ends of the target and the $6 \mathrm{~mm}$ section on the end of the target away from the spectrometer. First the conditions were arranged so as to reproduce the previous $10 \mathrm{~mm}$ spectra and then the emission from the two $2 \mathrm{~mm}$ sections at each end of the target was compared.
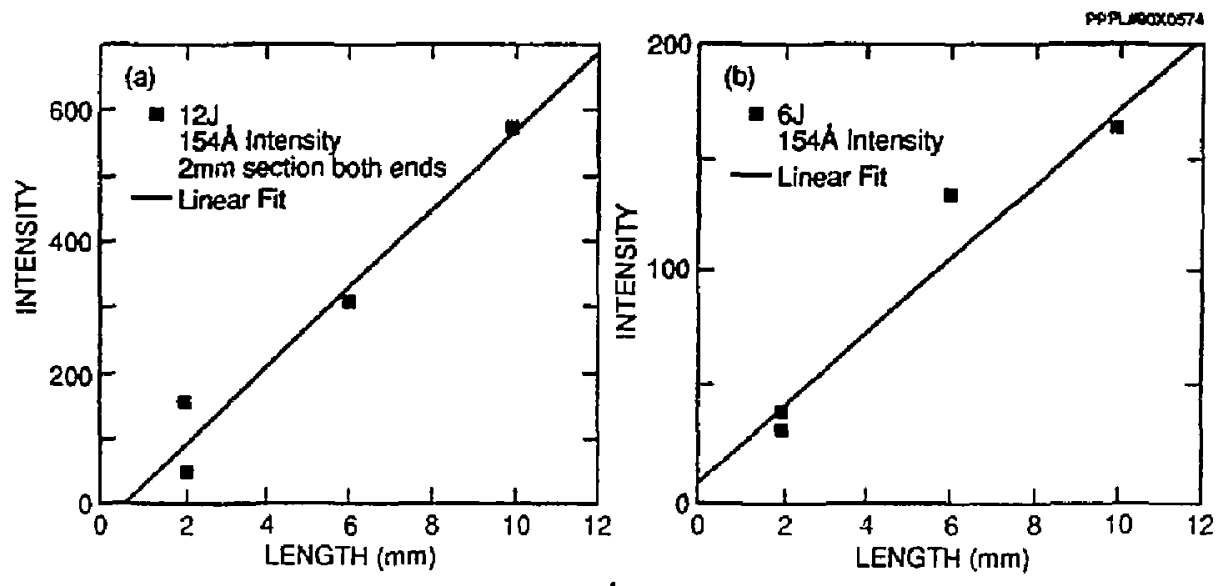

Fig. 6 (a) data showing the $154 \AA$ intensity yariation with length but in this case including $2 \mathrm{~mm}$ sections from both ends of the target. The $6 \mathrm{~mm}$ data was from a section at the opposite end of the target to the data in Figures 4 and 5 . (b) as above but with the target rotared by $2^{\circ}$. 

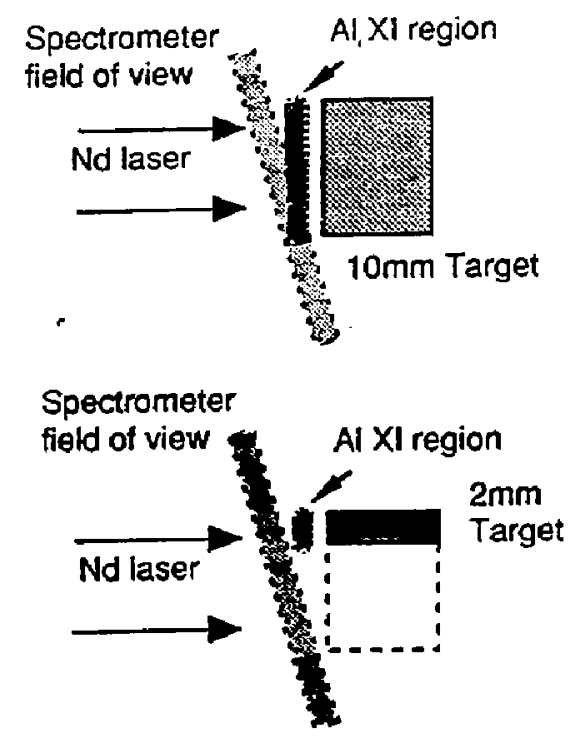

Fig. 7 Dlustration of the effect of a swall angle between the plasma and the region viewed by the spectrometer. The comparison of plasmas of differing lengths cannot be used for measurements of gain in this case.

It was immediately apparent that there was a dramatic difference in intensity between the $2 \mathrm{~mm}$ section at the spectrometer end and the $2 \mathrm{~mm}$ section at the opposite end. The target on one side of the laser beam looked much brighter than a target on the other side. Taking the average of the $2 \mathrm{~mm}$ results, the best fit wo the data was now a linear increase in intensity with length as shown in Fig. 6. The reason for the non-uniformity lay in a small angle between the target surface and the region viewed by the spectrometer (Fig. 7), possibly caused by refraction.

To verify this, the target was rotated about a vertical axis to change the position of the plasma generated by the two $2 \mathrm{~mm}$ sections with respect to the region viewed by the spectrometer. With a $2^{\circ}$ rotation the AlXI $154 \AA$ emission from the two ends became equal. In this configuration however the length dependence of the emission was linear. Basically the length scaling method to measure gain failed because the plasma was not homogeneous along the viewing region. In conclusion; the non-linear increase in Figs 4 and $S$ was caused by geometrical effects and not by stimulated emission.

As noted before, the measurement of an exponential intensity increase with length is commonly regarded as conclusive evidence for gain (see for instance Hara 1989). However in view of the above results it is clear that while this may be an encouraging sign of gain it is by no means sufficient proof that gain is present. As was done in some earlier works (for example Matthews 1985, Jamelot 1988, Keane 1989, and Kim 1989a), it is critical to monitor the emission from nearby spontaneous emission lines in the same ion, preferably lines with the same lower level as the lasing line, to be assured that one is viewing a homogeneous plasma and that the comparison of plasmas of differing lengths is a valid. Checking that the spontaneous emission lines have a linear length dependence is paricularly important for measurements of low gain-lengths, GL $\leq 4$, where the output intensities are not so different to spontaneous emission levels. 


\section{SUMMARY}

Soft $x$-ray laser technology is, at present, in a transition between demonstrations of gain in different systems and the acceptance of $x$-ray lasers as useful tools with practical applications. Good progress has been made in the application of soft $x$-ray lasers to microscopy and other fields. Gain at remarkably low pump energies has been demonstrated in CVI recombination systems and this augurs well for the development of low cost $x$-ray lasers for a variety of applications. Finally some piffalls in the measurement of gain in modest gain-length systems have been demonstrated.

\section{ACKNOWLEDGMENTS}

We would like to thank $\mathrm{E}$. Valeo for assistance with the gain fitting computer program and J. Schwarzmann, A. Schessler, D. DiCicco and G. Drozd for their technical support and laser operation. This work was supported by the U.S. Department of Energy, Advanced Energy Projects of Basic Energy Sciences.

\section{REFERENCES:}

Hara T, Ando K, Kusakbe N. Yashiro H and Aoyagi Y, 1989, Jpn. J of Appl. Phys. 6, 1010.

Kelly R L, 1987, J. Phys. Chem Ref. Data, 16, Suppl. 1

Kim D, Skinner C H, Umesh G, and Suckewer S, 195\%a, Opt . Lett ., 14, 665

Kim D, Skinner C H, Wouters A, Valeo E, Voorhees D, and Suckewer S, 1989b, J. OpR. Soc. Am. B 6, 115.

Jaegle $\mathrm{P}, 1990$, this proceedings

Jamelor G, Jaegle p, Carillon A, Gadi F, Gauthe B, Gennou H, Klisnick A, Möller C, and Sureau A, 1988, IEEE Trans. on Plasma Sci. 16, 497.

Keane C J, Ceglio N M, MacGowan B J, Matthews D L, P.ilson D G, Trebes J E, and Wilson D A, 1989, J. Phys. B. 22, 3343.

Linford $G J_{2}$ Peressini E R, Sooy W R, and Spaeth M L ,1974, Appl Opt 13, 379

Matthews D L et al. 1985, Phys Rev. Lett. 54, 110.

Schwob J L, Wouters A, and Suckewer S, 1987, Rev. Sci. Instrum. 58, 1601.

Skinner C H, DiCicco D S, Kim D, Rosser R J, Suckewer S, Gupta A P and Hirschberg j G, 1990a, Journal of Microscopy, 159, 51-60

Skinner C H, Kim D. Voortees D and Suckewer S, 1990b, J Opt Soc Am B 7, 2042

Suckewer S, 1990 This proceedings

Tighe B, 1990 This proceedings 